\title{
COMPARACIÓN ENTRE EL SISTEMA INTRAUTERINO DE LIBERACIÓN DE LEVONORGESTREL Y LA ABLACIÓN ENDOMETRIAL EN EL TRATAMIENTO DE LA HEMORRAGIA UTERINA DISFUNCIONAL
}

\author{
Laura Melado V. ', Mónica Novelle G. 1, Alicia Hernández G. 1, Mar Muñoz M. 1, Juan \\ Ordás ST. 1
}

1 Servicio de Ginecología y Obstetricia, Hospital Universitario La Paz, Madrid, España.

\section{RESUMEN}

Objetivo: Comparación entre el sistema intrauterino de liberación de levonorgestrel (SIL-LNG) y las técnicas de ablación endometrial (AR-E) en el tratamiento de la hemorragia uterina disfuncional (HUD). Método: Estudio retrospectivo de 170 pacientes con HUD distribuidas de la siguiente forma: 51 de ellas en el grupo AR-E y 119 en el grupo SIL-LNG. Se estudió el ciclo menstrual, la hemoglobina y el hematocrito, los efectos adversos y la satisfacción de las usuarias. Resultados: En ambos grupos se observó un incremento de los niveles de hemoglobina y hematocrito. El patrón de sangrado más frecuente fue el de amenorrea, hipomenorrea y ciclos normales tras los tratamientos. El grado de satisfacción fue muy elevado en ambos grupos. Conclusión: Ambos tratamientos son efectivos para mejorar la HUD. La paciente debe conocer los beneficios y riesgos de cada uno y elegir el más conveniente.

\section{PALABRAS CLAVE: Sistema intrauterino de liberación de levonorgestrel, ablación-resección endo- metrial, hemorragia uterina disfuncional}

\section{SUMMARY}

Objective: To compare the efficacy of levonorgestrel intrauterine system and endometrial resection in the treatment of dysfunctional uterine bleeding. Methods: Retrospective study with 170 women, 51 of them in endometrial resection group and 119 in the levonorgestrel intrauterine system group. Hemoglobin levels, menstrual bleeding patterns, adverse effects and degree of satisfaction were evaluated. Results: Hemoglobin levels increased in both groups. The most frequent bleeding patterns were amenorrhea, hypomenorrhea and normal pattern. The most of the patients in both groups were satisfied with the treatment. Conclusion: Both treatments were effective in reducing dysfunctional uterine bleeding. The woman must know risks and benefits from each one.

KEY WORDS. Levonorgestrel intrauterine system, endometrial resection, dysfunctional uterine bleeding 


\section{INTRODUCCIÓN}

La hemorragia uterina disfuncional (HUD) se define como aquella de origen uterino excesivo en intensidad y/o duración, no debida a patología orgánica pélvica o sistémica. Constituye una de las causas más frecuentes de consulta ginecológica, alcanzando hasta el $10 \%$ de las mismas. A pesar de que constituye una afección benigna, conlleva múltiples repercusiones sanitarias y un descenso en la calidad de vida de la mujer.

Para su manejo terapéutico, existen múltiples opciones: tratamientos médicos como antifibrinolíticos, AINEs, ácido mefenámico, anticonceptivos, progestágenos, danazol, análogos de $\mathrm{GnRH}$; sistema intrauterino de liberación mantenida de levonorgestrel (SIL-LNG); ablación-resección endometrial (AR-E); histerectomía; etc.

EI SIL-LNG realiza una acción local dentro de la cavidad uterina produciendo cambios atróficos en el endometrio, al disminuir la proliferación endometrial e incrementar la apoptosis en glándulas y estroma. A nivel molecular, este efecto está mediado por un aumento en la expresión del antígeno FAS y descenso de la proteína Bcl-2 y del antígeno nuclear de proliferación celular (1).

La AR-E consiste en una cirugía endometrial bajo control histeroscópico (ablación endometrial histeroscópica - HEA) con destrucción del endometrio hasta la capa basal. Puede realizarse con electrocirugía, láser-vaporización o rollerball. Su tasa de éxito alcanza el $90 \%$ disminuyendo el sangrado menstrual, con un riesgo de complicaciones del $4 \%$, que fundamentalmente consisten en perforación uterina (2). Hoy también se puede realizar ablación endometrial no histeroscópica (NHEA) mediante hidrotermia, radiofrecuencia, etc. Este tipo presenta resultados similares a largo plazo que el anterior, siendo una técnica más sencilla y con menores complicaciones intraquirúrgicas (3).

El objetivo de nuestro trabajo es la comparación entre la AR-E histeroscópica con el SIL-LNG como alternativas a la histerectomía en mujeres con HUD, valorando la efectividad, satisfacción y ventajas de cada uno de ellos en nuestro servicio.

\section{MATERIAL Y MÉTODO}

Estudio retrospectivo en el que se han incluido 170 mujeres premenopáusicas con HUD, recogidas desde enero de 2000 hasta enero de 2007; 51 de ellas en el grupo ablación y 119 en el grupo SILLNG. Los criterios de exclusión fueron: presencia de anomalías uterinas (congénitas o adquiridas), presencia de formaciones anexiales, citología cer- vicovaginal patológica, pólipo endometrial y mioma submucoso. A todas las pacientes se les realizó una revisión ginecológica previa con exploración física y ginecológica, ecografía, analítica y citología cervicovaginal.

En el grupo SIL-LNG (Mirena®), la inserción del dispositivo intrauterino (DIU) se realizó en consulta durante los cinco primeros días de la menstruación, con comprobación inmediata de la correcta colocación mediante ecografía vaginal y a las seis semanas, tras haber realizado la inserción. Se valoró ecográficamente el grosor endometrial previo a la inserción del DIU y a los tres meses de seguimiento, la presencia de sangrado intermenstrual (spotting) y la duración del mismo.

En el grupo AR-E, realizamos electrocirugía con resectoscopio empleando glicina al 1,5\% mediante bomba de infusión, tanto para la distensión de la cavidad uterina como para mantener dicha distensión. En este grupo, se analizaron las complicaciones intraoperatorias y en el postoperatorio inmediato, y el tiempo medio de estadía hospitalaria de las pacientes.

En ambos grupos se valoró el tipo de ciclo menstrual de la paciente antes y tras el tratamiento elegido (hipermenorrea, menorragia, polimenorrea, ciclos normales, oligomenorrea y amenorrea), el nivel de satisfacción de la usuaria (mejor, igual o peor respecto al estado previo al tratamiento), y un control analítico a los 6 meses de seguimiento con hemoglobina, hematocrito y ferritina.

El análisis estadístico se realizó mediante el programa Statistical Package Science v. 12.0 (SPSS 12.0 for Windows) con el cálculo de valores estadísticos descriptivos. Las diferencias en las proporciones se calcularon mediante la prueba de $\chi^{2}$ en las variables paramétricas y el test de Mann-Whitney para variables no paramétricas. Se aceptó un valor de significación estadística de $p \leq 0,05$ para las pruebas del estudio.

\section{RESULTADOS}

La distribución de ambos grupos fue homogénea tanto en la edad como en la paridad (Tabla I). Las pacientes con edad $\leq 45$ años tenían mayor frecuencia en el grupo SIL-LNG, mientras que en el grupo AR-E predominaban las de edad $>45$ años (Figura 1).

Las complicaciones quirúrgicas presentes en el grupo AR-E durante la realización del procedimiento fue de $4 \%$ (una perforación uterina que se resolvió con terapia antibiótica y la formación de una falsa vía que desencadenó una hemorragia profusa, resuelta mediante histerectomía). Durante 
el seguimiento posterior de este grupo, el $4 \%$ de las pacientes precisaron una segunda ablación por recidiva clínica y $8 \%$ (cuatro pacientes del total) requirieron histerectomía post-ablación, una de ellas por el resultado anatomopatológico de hiperplasia atípica del tejido endometrial resecado y tres por HUD recidivante.

\section{Tabla I}

\section{CARACTERÍSTICAS DE AMBOS GRUPOS}

\begin{tabular}{lcc}
\hline \multicolumn{1}{c}{ Variables } & AR-E & SIL-LNG \\
\hline Edad (años) & $43 \pm 5$ & $42 \pm 4$ \\
Paridad ( $n^{\circ}$ hijos) & $2 \pm 0,9$ & $2 \pm 0,9$ \\
\hline
\end{tabular}

AR-E: ablación-resección endometrial. SIL-LNG: sistema intrauterino de liberación mantenida de levonorgestrel.

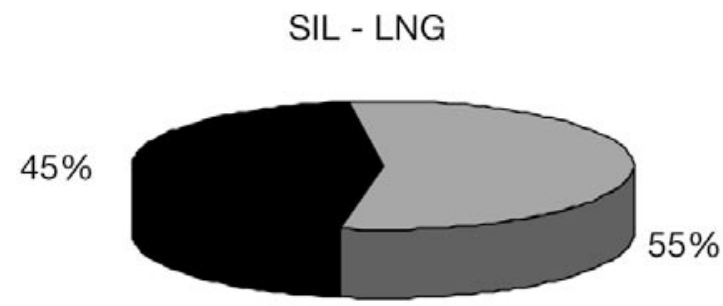

5 y 6). Tampoco se hallaron diferencias significativas por grupos de edad ( $\leq 45$ años/>45 años) en la mejoría del control del ciclo. No obstante, aparece una mayor tasa de amenorreas en el subgrupo de pacientes $>45$ años en las que se realizó AR-E que en el grupo SIL-LNG (31\% vs 19\%) siendo esta diferencia estadísticamente significativa $(p<0,05)$.

En las pacientes del grupo AR-E, el tiempo medio de estadía hospitalaria fue de 32,4 horas.

En el grupo SIL-LNG se constató un descenso del grosor endometrial a los tres meses de tratamiento, medido con ecografía vaginal (Figura 7). La aparición de spotting fue de $2 \pm 1,4$ meses tras la inserción del DIU, y hasta un $9,3 \%$ de las pacientes refirieron algún efecto secundario, siendo los más frecuentes tensión mamaria y cefalea.

El nivel de satisfacción que presentaron las pacientes fue alto en ambos grupos, ligeramente

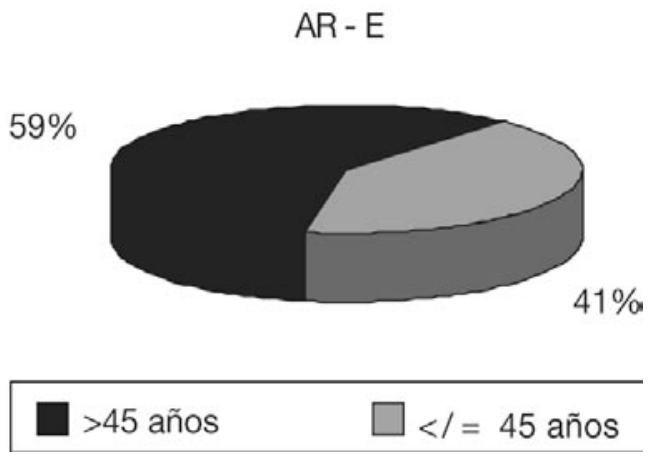

Figura 1. Distribución por edades del tratamiento. AR-E: ablación-resección endometrial. SIL-LNG: sistema intrauterino de liberación mantenida de levonorgestrel.

En el grupo SIL-LNG, una paciente presentó pérdida espontánea del DIU a los pocos días tras su colocación. En el 3,4\% de las pacientes de este grupo no se pudo realizar la inserción del dispositivo al no conseguir canalizar el cérvix. Por otro lado, el $1,7 \%$ de ellas precisaron otro tratamiento posterior por insatisfacción o HUD recidivante.

Al estudiar los resultados analíticos observamos una mejoría significativa en las tasas de hemoglobina y hematocrito con cada tratamiento y al evaluar el global de pacientes $(p<0,0001)$, si bien no encontramos diferencias estadísticamente significativas entre ambas técnicas (Figuras 2 y 3 ).

Estudiando el tipo de ciclo en el global de las pacientes, se produjo una mejoría significativa $(p<0,0001)$, es decir, la mayoría presentaban ciclos normales, oligomenorreas o amenorrea tras el tratamiento (Figura 4). Al desglosarlas por grupos de tratamiento, no aparecieron diferencias estadísticamente significativas entre ambas técnicas (Figuras mayor en el grupo SIL-LNG, sin que las diferencias fuesen significativas (Figura 8).

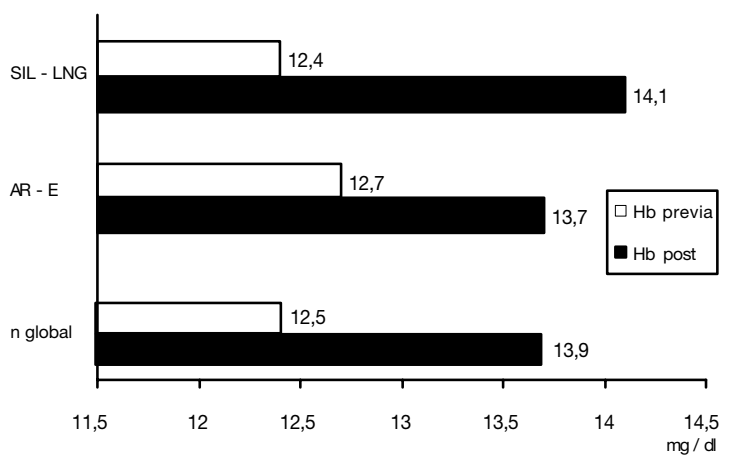

Figura 2. Hemoglobina $(\mathrm{Hb})$ previa al tratamiento y posterior. AR-E: ablación-resección endometrial. SIL-LNG: sistema intrauterino de liberación mantenida de levonorgestrel. 


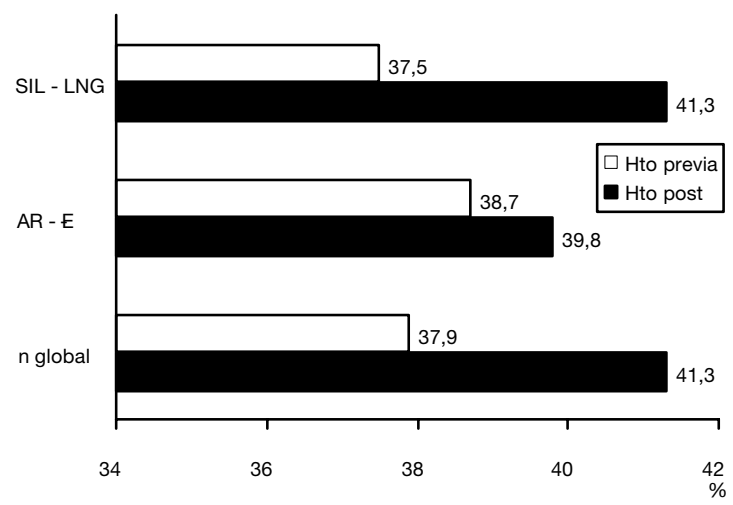

Figura 3. Hematocrito (Hto) previo al tratamiento y posterior. AR-E: ablación-resección endometrial. SIL-LNG: sistema intrauterino de liberación de levonorgestrel.

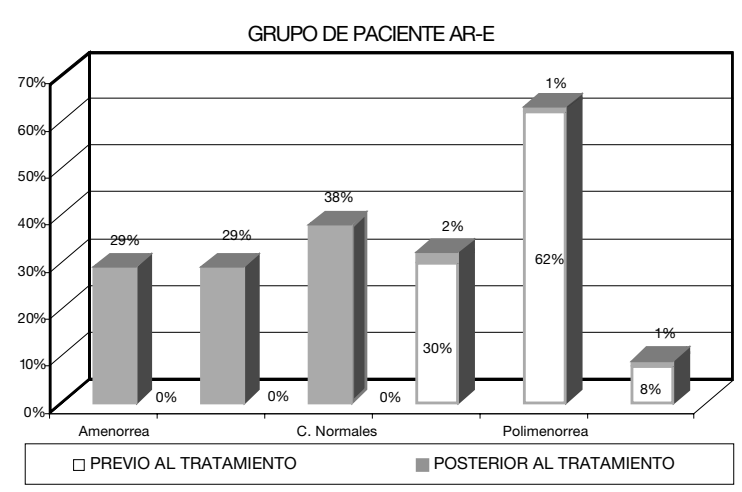

Figura 5. Tipo de ciclo previo y posterior al tratamiento que presentaban las pacientes del grupo AR-E (ablación-resección endometrial).

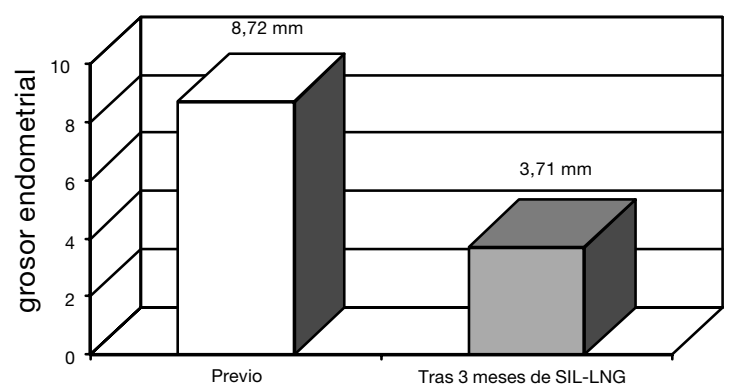

Figura 7. Grosor endometrial, valorado mediante ecografía vaginal, que presentaron las pacientes antes y tras meses tras el tratamiento con SIL-LNG (sistema intrauterino de liberación mantenida de levonorgestrel).

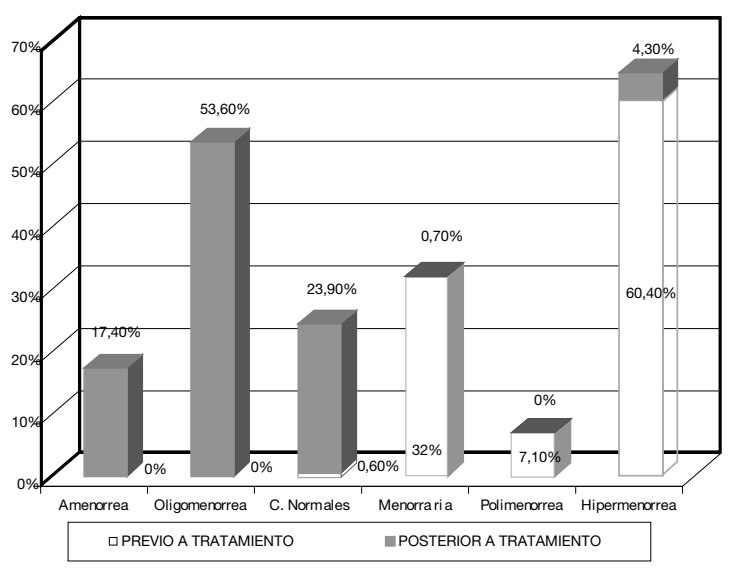

Figura 4. Tipo de ciclo del global de pacientes previo y posterior al tratamiento.

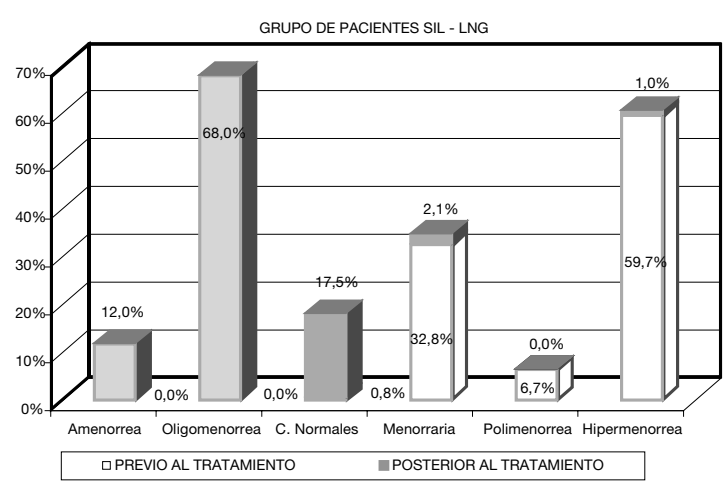

Figura 6. Tipo de ciclo previo y posterior al tratamiento que presentaban las pacientes del grupo SIL-LNG (sistema intrauterino de liberación mantenida de levonorgestrel).

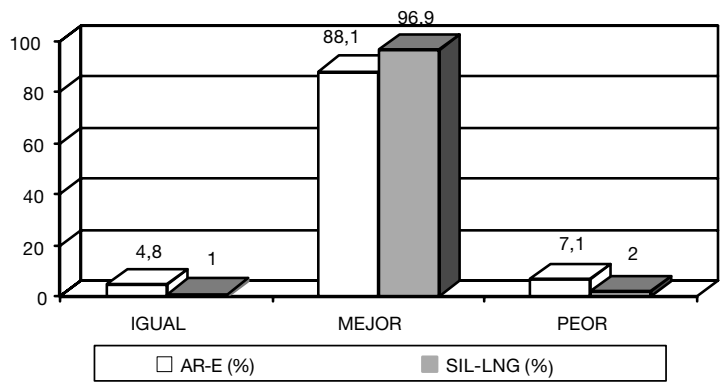

Figura 8. Satisfacción de las usuarias. AR-E: ablación-resección endometrial. SIL-LNG: sistema intrauterino de liberación mantenida de levonorgestrel. 


\section{DISCUSIÓN}

A la vista de estos resultados, tanto la AR-E como la utilización de SIL-LNG son eficaces en el tratamiento de la hemorragia uterina disfuncional. Con ambas técnicas se consigue incrementar los valores plasmáticos de hemoglobina y hematocrito así como mejorar el patrón de sangrado menstrual, ya que en un alto porcentaje de nuestras pacientes, los ciclos que presentaron tras el tratamiento seguían patrones de amenorrea/oligomenorrea. Además, el grado de satisfacción de las mujeres es muy elevado en ambos grupos (3-9).

EI SIL-LNG es un método poco agresivo, de colocación ambulatoria y que presenta complicaciones menores derivadas de la técnica de inserción de un DIU, sin precisar ingreso ni cirugía. Además, es un tratamiento reversible, que permite preservar la fertilidad, por lo cual puede ser una buena alternativa en aquellas pacientes de menor edad con clínica de menorragia y en las que una técnica definitiva puede no ajustarse a sus deseos.

La AR-E presenta mayores costos derivados tanto de la estancia hospitalaria como del procedimiento, sin olvidar el riesgo quirúrgico para la paciente. Sin embargo, ofrece la posibilidad de realizar un estudio histológico del endometrio y una mayor tasa de amenorrea, hecho que puede interesar especialmente a ciertos grupos de pacientes que deseen un tratamiento definitivo.

Por tanto, dado que ambos procedimientos son de una eficacia elevada, consideramos que las pacientes deben recibir una información detallada sobre los beneficios y riesgos de ambas técnicas, y deben tener una actitud activa en la elección del tratamiento que más se ajuste a sus necesidades personales, si ha cumplido o no sus deseos genésicos o si desea una técnica más agresiva por insatisfacción de tratamientos previos. Ambos tra- tamientos no son excluyentes el uno del otro, por lo que su elección puede ser secuencial en función de la satisfacción de la paciente.

\section{BIBLIOGRAFÍA}

1. Maruo T, Laoag-Fernandez JB, Pakarinen $\mathrm{P}, \mathrm{Mu}-$ rakoshi $\mathrm{H}$, Spitz IM, Johansson E. Effects of the levonorgestrel-releasing intrauterine system on proliferation and apoptosis in the endometrium. Hum Reprod 2001;16(10):2103-8.

2. O'Connor H, Magos A. Endometrial resection for the treatment of menorrhagia. N Engl J Med 1996;335(3):151-6.

3. Marjoribanks J, Lehtaby A, Parquhar C. Surgery versus medical therapy for heavy menstrual bleeding. Cochrane Database Syst Rev, 2003 (2), p.CD00385.

4. Pakarinen $p$, Luukkainen T. Five years' experience with a small intracervical/intrauterine levonorgestrelreleasing device. Contraception 2005;72(5):342-5.

5. Lethaby A, Cooke I, Rees M. Progesterone or progestogen-releasing intrauterine systems for heavy menstrual bleeding. Cochrane Database Syst Rev. 2005 Oct 19;(4):CD002126.

6. Banu NS, Manyonda IT. Alternative medical and surgical options to hysterectomy. Best Pract Res Clin Obstet Gynaecol 2005;19(3):431-49.

7. Bourdrez P, Bongers MY, Mol BW. Treatment of dysfunctional uterine bleeding: patient preferences for endometrial ablation, a levonorgestrel-releasing intrauterine device or hysterectomy. Fertil Steril 2004;82(1):160-6.

8. Busfield RA, Farquahar CM, Sowter Mc, Lethaby A, Sprecher M, Yu Y, et al. A randomised trial comparing the levonorgestrel intrauterine system and thermal balloon ablation for heavy menstrual bleeding. BJOG 2006; 113(3): 257-263.

9. Lázaro J, de la Fuente P, Montoya L, Repollés M. Resección endometrial y sistema intrauterino de liberación de levonorgestrel en el tratamiento de la hemorragia uterina disfuncional. Prog Obstet Ginecol 2003;46(9):377-81. 